\title{
Promoter activity dynamics in the lag phase of Escherichia coli
}

Daniel Madar ${ }^{1}$, Erez Dekel ${ }^{1}$, Anat Bren ${ }^{1}$, Anat Zimmer ${ }^{1}$, Ziv Porat ${ }^{2}$ and Uri Alon ${ }^{1 *}$

\begin{abstract}
Background: Lag phase is a period of time with no growth that occurs when stationary phase bacteria are transferred to a fresh medium. Bacteria in lag phase seem inert: their biomass does not increase. The low number of cells and low metabolic activity make it difficult to study this phase. As a consequence, it has not been studied as thoroughly as other bacterial growth phases. However, lag phase has important implications for bacterial infections and food safety. We asked which, if any, genes are expressed in the lag phase of Escherichia coli, and what is their dynamic expression pattern.

Results: We developed an assay based on imaging flow cytometry of fluorescent reporter cells that overcomes the challenges inherent in studying lag phase. We distinguish between lag1 phase- in which there is no biomass growth, and lag2 phase- in which there is biomass growth but no cell division. We find that in lag1 phase, most promoters are not active, except for the enzymes that utilize the specific carbon source in the medium. These genes show promoter activities that increase exponentially with time, despite the fact that the cells do not measurably increase in size. An oxidative stress promoter, katG, is also active. When cells enter lag2 and begin to grow in size, they switch to a full growth program of promoter activity including ribosomal and metabolic genes.

Conclusions: The observed exponential increase in enzymes for the specific carbon source followed by an abrupt switch to production of general growth genes is a solution of an optimal control model, known as bang-bang control. The present approach contributes to the understanding of lag phase, the least studied of bacterial growth phases.
\end{abstract}

Keywords: E. coli, Lag phase, Resource allocation, Optimal control theory, Bang-bang control, Pontryagin maximum principle, Transcriptional program, Stringent response

\section{Background}

When bacteria are inoculated into fresh medium, they often show a period without growth known as the lag phase [1-3]. Lag phase is interesting as a fundamental biological process in which bacterial physiology adapts to a new environment. Lag phase is also of interest in fields such as food safety- in which lag phase is one factor in determining food shelf life [4-6]. In medicine, lag phase plays a role when bacteria move into the blood stream or other locations where rapid growth can occur. In both food safety and medicine, the longer the lag phase the longer body defenses have time to fight pathogens, and the longer the natural gut flora have to out-compete the pathogens. In some cases, longer lag phase is associated

\footnotetext{
*Correspondence: uri.alon@weizmann.ac.il

'Department of Molecular Cell Biology, Weizmann Institute of Science, Rehovot 76100, Israel

Full list of author information is available at the end of the article
}

with increased invasiveness of bacterial cells $[7,8]$, or with antibiotic tolerance [9].

There are several definitions of lag phase [2-4,10-18] -See Additional file 1: Table S1 for details. For clarity, we divide lag phase into two sub-phases: lag1 is the period between inoculation and until biomass begins to grow, and lag2 is the period between the end of lag1 and the time that cell numbers begin to increase (Figure 1A, B and Additional file 1: Figure S1). Lag1 has also been called the adaptation phase [13], a term we do not use because of the multiple meanings of adaptation in sensing and evolution. Note that lag1 is not adaptation by mutation, but rather acclimatization of the cell physiology to new conditions. The name 'lag2' is contradictory in the sense that it is a phase of growth, namely the first cell generation. We however chose this name to describe the second part of the lag phase because cells do not grow in number, which

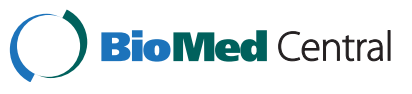


A

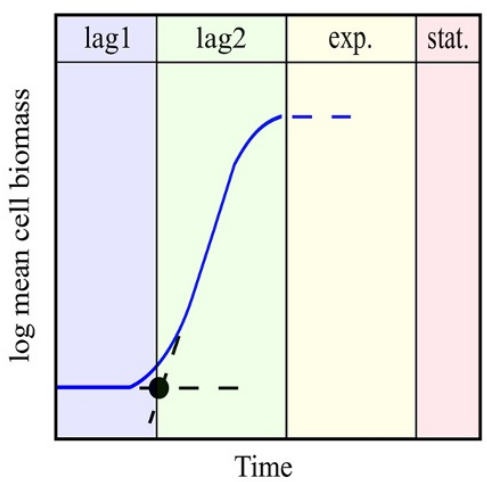

B

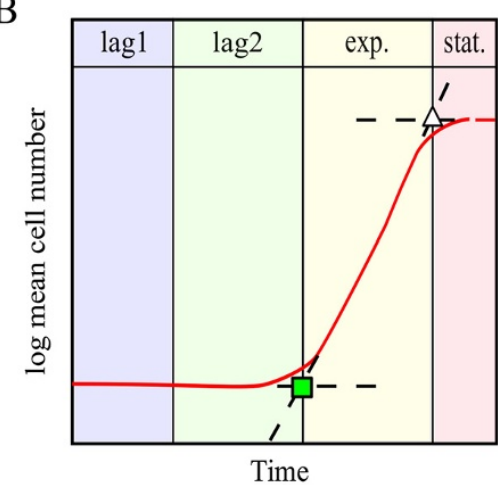

C

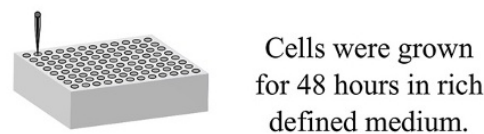

1:750 dilution

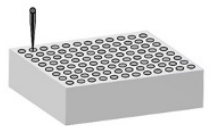

Cells were grown in medium of choice

for various amounts of time.
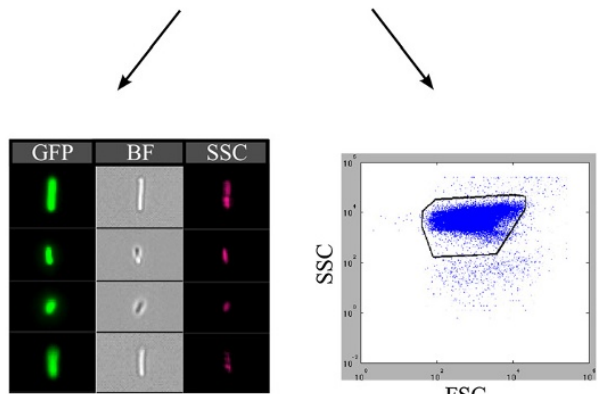

FSC

Cells were analysed using standard and imaging flow cytometry.

Figure 1 Definition of lag1 and lag2 phases, and the experimental system. (A) A schematic representation of mean cell biomass over time, with separation into phases. In lag1, biomass does not measurably increase. The end of lag1 is operationally defined using the biomass (or cell length) curve, at the intersection between the constant biomass line at the beginning of the curve, with the extrapolated exponential increase in biomass in lag2 (black circle). In lag2, biomass increases. The end of lag2 is defined by the first division events. At this point biomass plateaus (or peaks and then declines), as cells reach a biomass characteristic of exponential growth. See Additional file 1: Table S1, and Figure S1.

(B) A schematic representation of mean cell number over time, with separation into phases. The end of lag2 is operationally defined by the intersection between the constant cell number line at the beginning of the curve, with the extrapolated exponential increase in cell number (green rectangle). The end of exponential phase is operationally defined by the intersection between the increasing cell number line, with the constant cell number line at the end of the curve (white triangle). See Additional file 1: Table S1, and Figure S1. (C) Schematic description of the experiment: cells were grown for 48 hours in rich defined medium, inoculated into poor or rich defined medium, grown for various amounts of time in a robotic system, and analyzed by standard flow cytometry and by imaging flow cytometry.

is why previous works considered this phase to be part of lag phase.

Operationally, the length of lag1 phase is determined in a logarithmic plot of biomass or a related quantity (cell length, optical density, dry weight) by extrapolating the exponential growth of biomass and finding its intersection with the initial biomass (Figure 1A). The end of lag2 can be similarly defined from a logarithmic graph of cell numbers (Figure 1B).

When cells are grown overnight in a rich medium and then transferred to a poor medium, both lag1 and lag2 phases may occur. Mathematical models of lag phase include Baranyi's model [19] in which biomass production relies on accumulation of bottleneck proteins in lag phase $[18,19]$, and models which focus on cell-cell variation in lag phase duration $[4,20]$. Each individual cell has lag1 and lag2 phases, but the duration of these phases varies stochastically between individual cells, as is evident in the measurements of Métris et al. [21] (Additional file 1: Figure S1). One possible origin of these cell-cell variations is stochastic variation in the levels of enzymes present in the cell [22-25].

Individual cell measurements also show that the cell cycle accelerates during the first few divisions $[20,21,26]$, and thus it takes several generations to reach the maximal exponential growth rate. Thus, lag2 as defined here is only the first generation in a prolonged acceleration phase [3] (Additional file 1: Figure S1).

Studies of gene expression in bacterial lag phase are relatively few [27-29]. Several studies have focused on particular genes, such as the transcription regulator fis [30] and ribosomal genes [31-33]. A recent study explored the 
genome-wide expression patterns of Salmonella Typhimurium upon transition from stationary phase in rich medium (48 hours growth in LB) to fresh LB medium [34]. In this case, bacteria seem to make biomass almost immediately (lag2), making it difficult to resolve lag1 phase. The expression program in lag2 was similar to expression in exponential growth, with some differences in genes related to metal ion accumulation, phosphate accumulation and oxidative stress. Thus, current knowledge is greatest for lag2, the phase in which biomass grows, suggesting that it is similar in many ways to exponential phase.

In contrast to lag2, there is little knowledge about lag1 phase, the phase in which biomass does not grow. This lack of knowledge is due in part to the technical difficulty of studying lag1, and in part to the fact that commonly used protocols go from rich medium to rich medium so that lag1 is very short. It is therefore not clear whether the expression program in lag1 is fundamentally different from the expression in lag2 and exponential growth. For example, one may imagine several possibilities: either no expression in lag1 of any gene, expression that is directed only towards selected genes, or expression of the same genes as in exponential phase but at low intensity.

Here, we address the nature of lag phase by developing an assay for measuring cell size, cell number and promoter activity at high accuracy in individual bacterial cells in lag1 and lag2 phases. This assay allows us to overcome the problem of low signal to background that occurs in culture-based assays of lag phase that rely on fluorimeters for optical density (OD) and fluorescence measurements. We found that the expression program in lag1 phase is very different from that in lag2 and exponential phases. Most promoters are not measurably active in lag1 phase. The promoters of the utilization operons for the specific carbon source in the medium are, however, highly active in lag1 phase showing an exponential increase with time, despite the fact that biomass does not measurably increase. In lag2 and exponential phase, utilization genes, ribosomal genes and a wide range of growth genes are expressed together. This suggests a 'bang-bang' control strategy, known from optimal control theory in engineering: first concentrate resources on generating utilization genes for the specific carbon source, so that carbon can flow into energy production and building blocks (such as amino acids), and only then make ribosomes to produce biomass. The seemingly inert lag1 phase thus shows selective and strong expression of specific genes.

\section{Results}

Flow cytometry assay on fluorescent reporter cells allows differentiation between bacterial lag1 and lag2 growth phases

To measure promoter activity, we used strains from the E. coli reporter strain library previously developed in our lab [35-37]. Each strain bears a low-copy plasmid with fast folding GFP under the control of a full length copy of the promoter of interest.

A technical challenge for studying lag1 is low cell density: one cannot use the reporter strains to measure promoter activity during lag1 phase in a culture using a multi-well fluorimeter, because cell density and fluorescence is below the background detection limit [4,38]. One can use a high inoculum level (low dilution of stationary phase starters) to bypass the OD detection threshold, but this can affect lag phase duration and behavior due to the relatively high concentration of stationary phase stimulatory and inhibitory molecules [39-41], and due to quorum sensing [42-44]. Thus, we developed a flow cytometry assay to measure fluorescence and cell size from individual cells grown in batch culture (Figure 1C).

To prepare the cells for flow cytometry at multiple time points after inoculation, we used a robotic liquid handling system to transfer cells from stationary phase culture to 96- deep well plates in a time-delayed manner. Wells were inoculated at varying temporal intervals, ranging from 5 to 180 minutes. At the end of this procedure, the multi-well plate had cells grown in fresh medium for different amounts of time post inoculation, ranging from zero to 15 hours. Cells were kept on ice and measured in flow cytometers, so that samples represented a post-inoculation time course.

We used flow cytometry to measure cell number and individual cells' fluorescence. By viewing individual cells, flow cytometry overcomes the signal to noise problems of low density batch cultures. We measured total cell density by the number of cytometry counts at a given time point. This measurement of cell density agreed very well with colony-forming-unit measurements on agar plates (Additional file 1: Figure S4).

We also measured cells in an imaging flow cytometer, which provides images of individual cells. These images allowed a direct measurement of cell length. We used cell length to operationally define the end of lag1 phase, at the intersection between the initial cell length line at the beginning of the curve, with the extrapolated exponential increase in cell length in lag2 (Figure 1A, black circle). The end of lag2 is operationally defined using the cell number curve: it is the intersection of the cells' exponential growth line with the line of initial cell number (Figure 1B, green rectangle). The end of exponential phase is similarly defined as the intersection between the exponential growth line and the line of final cell density (Figure 1B, white triangle).

We studied cells transferred from a stationary phase culture in defined M9 medium with glucose $(0.2 \%)$ and casamino acids (M9CGLU), into fresh M9 medium with no casamino acids in which a given sugar (arabinose) is the sole carbon source at saturating $(0.2 \%)$ concentrations 
(M9ARA). As a control, we also studied cells inoculated into fresh M9CGLU. We found that for M9ARA, a significant lag1 phase was seen lasting for $3 \pm 1$ hours (Figure 2A). After the lag1 period, cells began to grow in length and entered lag2. The duration of lag2 was $5.4 \pm 1.2$ hours. Thus lag1 + lag2 lasted for $8.4 \pm 1.2$ hours. When cells entered exponential phase, the rise in cell numbers coincided with the plateauing of mean cell length (Figure 2B and Figure 2A respectively). This is because cell divisions prevent a further rise in mean cell length. The exponential growth rate was $0.76 \pm$ 0.07 hour $^{-1}$, corresponding to a generation time of $55 \pm$ 5 minutes.

In contrast, transfer to the richer medium with glucose and amino acids (M9CGLU) showed immediate growth in cell size, and thus cells entered lag2 without a measurable delay (Figure 2C). Here, the rise in cell number is also coupled to the peak of mean cell size (Figure 2D and Figure $2 \mathrm{C}$ respectively). The duration of lag2 was $2.2 \pm 0.5$ hours, the exponential growth rate is $1.4 \pm$ 0.4 hour $^{-1}$, and the exponential generation time was $29 \pm 8$ minutes. When cells entered stationary phase, their mean length decreased back to its pre-inoculation level (Figure 2C).
In lag1 phase, genes for arabinose utilization are expressed exponentially with time despite no growth in biomass

We measured the fluorescence of the reporter cells for genes related to arabinose utilization in fresh M9ARA medium. We found that the fluorescence of the arabinose utilization genes rises exponentially with time during lag1 and lag2 phases. The reporters for the catabolic operon $\operatorname{araBAD}$, and the transporters araE and $\operatorname{araFGH}$, show an exponential rise in fluorescence despite no measurable increase in biomass in lag1. araBAD expression rose by a factor of about 60 over the eight hours of lag1 + lag2, (Figure 3A), corresponding to an exponential rate of $0.45 \pm 0.02$ hour $^{-1}$ and a doubling time of $92 \pm 4$ minutes. Similarly, ara $E$ showed an exponential increase of about 60 fold, at rate $0.44 \pm 0.03$ hour $^{-1}$. This doubling time is nearly twice the cell doubling time of $55 \pm 5$ minutes in exponential phase in the same medium. As cells leave lag2 phase and begin to divide, these reporters reach a steadystate of fluorescence levels, because cell divisions dilute the GFP produced by these promoters.

One possible explanation for the exponential rise in reporter fluorescence during lag1 is an auto-catalytic process in which cells devote their gene expression resources to utilizing the sugar; the more transporters
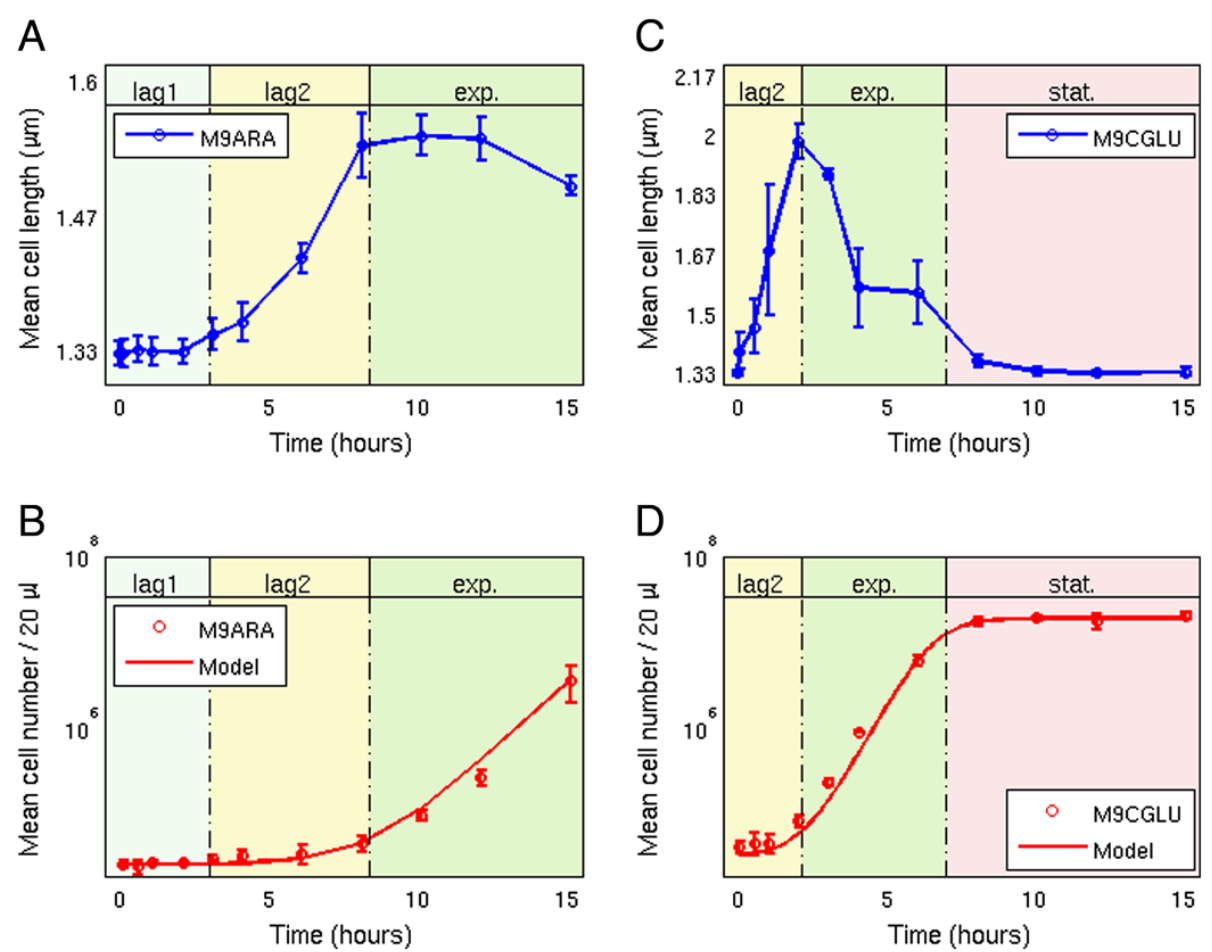

Figure 2 Cell length and cell number measurements allow growth curve segmentation into lag1 and lag2 phases. (A) Mean bacterial length in minimal arabinose medium (M9ARA). $1 \mu \mathrm{m}=3$ pixels. The blue line is a guide to the eye. (B) Mean cell numbers (in $20 \mu \mathrm{l}$ sample) in M9ARA medium. The red line is a fit to the optimal control model. (C) Mean bacterial length in minimal glucose + amino-acid medium (M9CGLU). $1 \mu \mathrm{m}=3$ pixels. The blue line is a guide to the eye. (D) Mean cell number (in $20 \mu \mathrm{l}$ sample) in M9CGLU medium. The red line is a fit to the model. Error bars indicate standard error. 

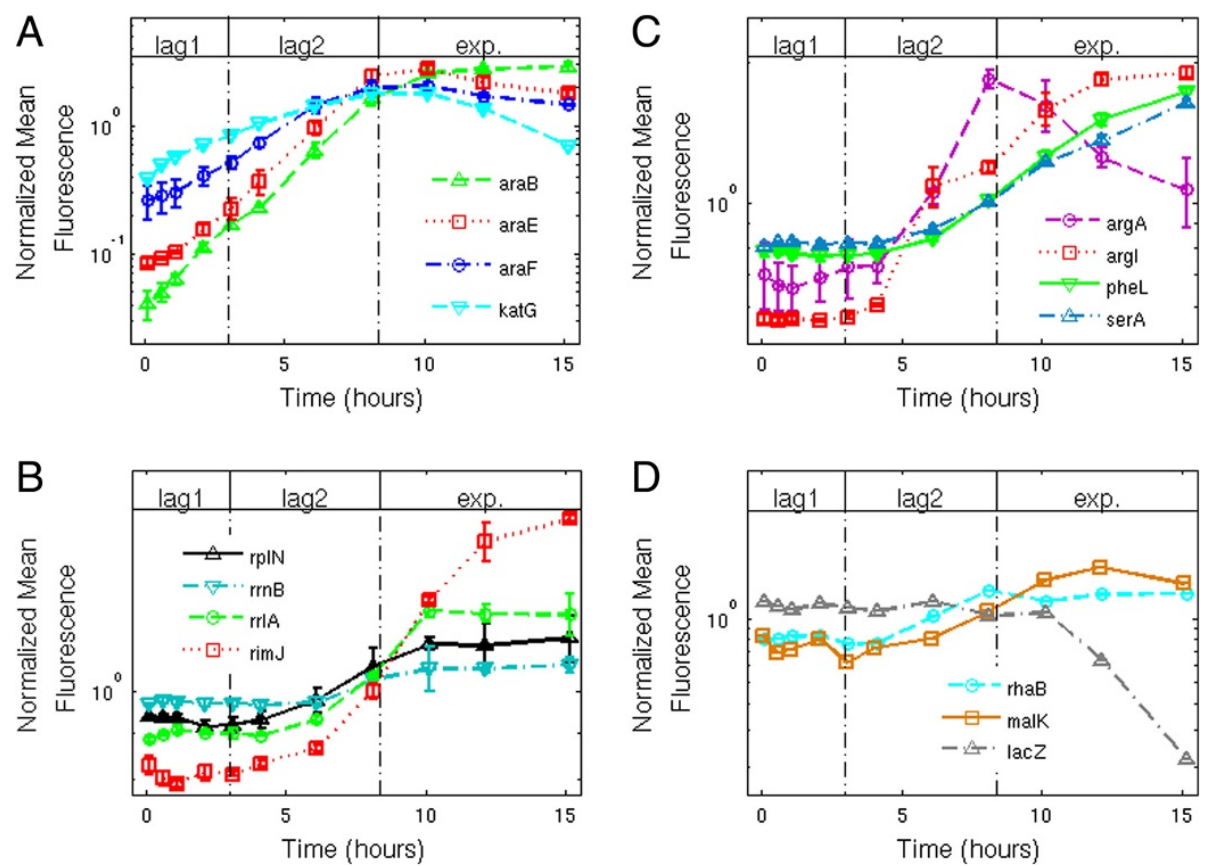

Figure 3 On arabinose minimal medium, the arabinose promoters are selectively activated during lag1. Mean GFP fluorescence of the cells in minimal arabinose medium (M9ARA), for different reporter strains. Growth phases are indicated. Data for each reporter was normalized by its mean value. Lines are guides to the eye. Error bars indicate standard error. (A) Normalized mean fluorescence of the arabinose system reporters, and of katG. See Additional file 1: Figure S3. (B) Normalized mean fluorescence of ribosomal genes reporters. (C) Normalized mean fluorescence of amino-acid genes reporters. (D) Normalized mean fluorescence of the rhamnose, maltose and lactose system reporters.

available, the more the sugar can be utilized giving rise to more expression and so on.

Individual cell data from the flow cytometry experiment indicates that the expression of the arabinose system promoter increases with time for all cells, and is not an all-or-none phenomenon in which some cells express the operons and others do not (Additional file 1: Figure S2).

In lag1 phase, growth genes are not measurably expressed We also tested ribosomal promoter activity in M9ARA medium, using relevant reporter strains $(r p l N, r r n B, r r l A$, rimJ). We found that ribosomal reporters did not show an appreciable rise during lag1 phase in arabinose (Figure 3B). When cells began to grow in size (lag2 phase), the ribosomal promoters were activated (Figure $3 \mathrm{~B}$ ). This lag2 increase appears mild in the case of $r r n B$ due to its relatively high initial level. The promoters began to approach a steady-state activity levels in exponential phase, when their mean GFP production rates are balanced by the dilution rate due to cell division.

We also tested amino-acid biosynthesis promoters for arginine, phenylalanine and serine ( $\operatorname{argA}$, $\operatorname{argI}$, pheL and $\operatorname{ser} A$ ). We found that these promoters were not measurably active during lag1 on arabinose (Figure 3C), even though the medium did not contain amino acids. These promoters became active only in lag2, rising at different rates, approaching a steady-state in exponential phase.
We tested catabolic genes for other sugars, rhamnose, maltose and lactose (rhaB, malK and lacZ). We found that these promoters were also not measurably active in lag1 or thereafter (Figure 3D). Thus, cells seemed to selectively express the utilization genes for the nutrient in the medium (arabinose) in lag1, and not utilization genes for other sugars.

To extend our survey of promoter activity in lag1 phase, we performed a screen using 140 additional promoters, with diverse biological functions, in M9ARA medium. The promoters controlled genes involved in translation, transcription, global regulators, amino acid biosynthesis, oxidative stress, phosphate and metal transport and metabolism, cold shock, heat shock and global stress (see Additional files 1, 2 and 3). To allow a large screen, we used fewer time-points: three in lag1 and one in lag2 phase. No growth related promoters (ribosomal, transcriptional, translational, amino-acid biosynthesis, etc.) showed a measurable promoter activity. Genes with a significant increase in lag1 were re-tested at higher temporal resolution. We find that only one promoter reproducibly showed more than a 3fold increase during lag1, the oxidative stress promoter $k a t G$ (Figure 3A). However, it does not rise as rapidly as the arabinose promoters. We also found a minor increase in some of the iron metabolism and transporter promoters (see Additional files 2 and 3).

When cells were inoculated into glucose and amino acid medium (M9CGLU medium), instead of arabinose, they 
skipped lag1 and entered lag2 immediately. We found that the arabinose utilization promoters were not active during lag2 or exponential phases, and slightly rose at stationary phase when glucose is depleted and cAMP rises (Figure 4A). Ribosomal promoters were active immediately after inoculation, during lag2 and exp. phase (Figure 4B). The amino-acid promoters were mostly activated towards the end of the exp. phase, when the medium ran out of amino-acids (Figure 4C).

\section{Results are consistent with optimal control principle of 'bang-bang' control}

Taken together, these results suggest that in lag1 on arabinose, cells mostly focus their promoter activity on the arabinose utilization genes. They do not activate the vast majority of promoters, including ribosomal genes, amino acid biosynthesis genes or utilization genes for other sugars.

This is analogous to the control theory strategy known as bang-bang control. A bang-bang strategy posits that in order to produce a given amount of output in minimal time, invest first in producing the limiting component only, and then switch abruptly to making the other elements in the system. This is in contrast to a different strategy in which one makes all components at a given ratio throughout time. In lag1, cells devote their resources to making specific utilization proteins, and then, in lag2, switch to making ribosomes together with more utilization genes. To ask whether this strategy is optimal for making the most cells at a given time $T$ after inoculation, we analyzed a mathematical model based on Baranyi's model for bacterial growth [19]. The model, outlined in Figure 5, provides equations for the production of a bottleneck enzymes $P$ which provide the substrates for producing biomass $M$ (more details in the Methods section). One seeks the best way to temporally allocate production resources between making $P$ and making $M$, in order to double the biomass in minimal time.

The model can be exactly solved, using an optimal control method known as Pontryagin's maximum principle $[45,46]$. The exact solution shows that the optimal allocation is to first make only $P$ - as in lag1, and then switch to making both $P$ and $M$ - as in lag2 (Figure 5A-C). This conclusion applies to a wide range of models and parameters and is not sensitive to the details of the equations used [46].

The exact solution (Figure 5 and Methods section) suggests that the lag phase duration depends on the initial level of bottleneck enzyme $P_{O}$. The smaller $P_{O}$, the longer the lag phase because it is advantageous to spend time to produce $P_{O}$ in order to increase the subsequent rate of biomass production (more precisely, lag duration depends on $P_{O}$ logarithmically: $\left.\tau \sim \ln \left(1 / P_{0}\right)\right)$. If $P_{O}$ exceeds a critical amount, $P_{O C}$, it no longer becomes optimal to have a lag1 phase the optimal $\tau$ equals zero (Figure 5D). Instead, the cells

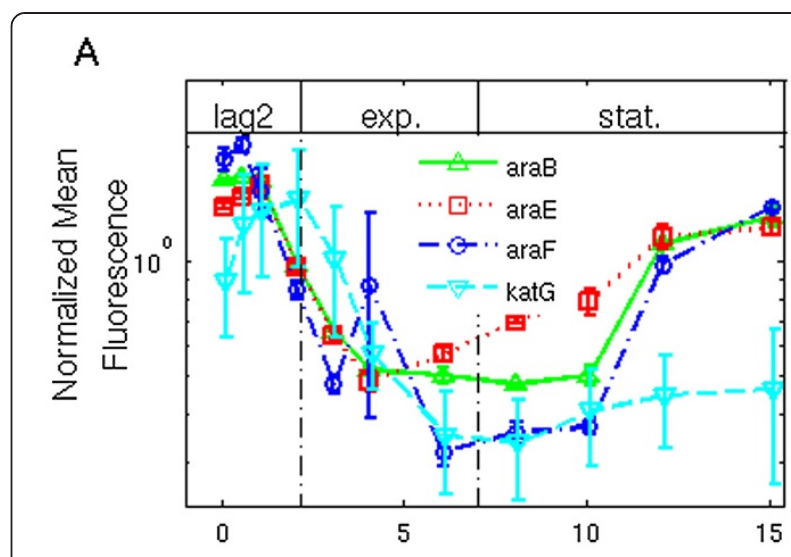

B

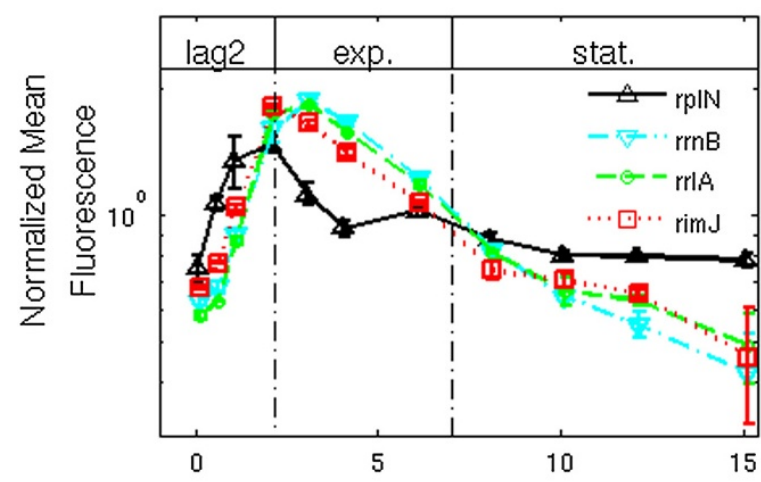

C

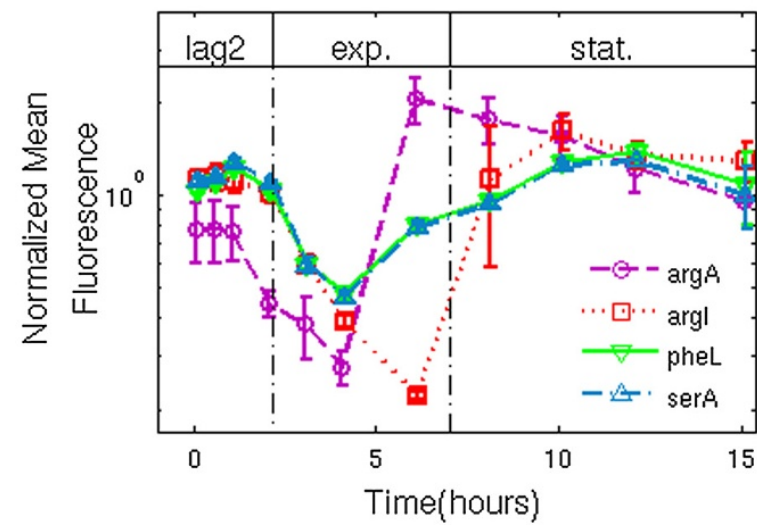

Figure 4 Cells enter lag2 immediately on glucose minimal medium, and express ribosomal promoters. Normalized mean GFP fluorescence of the cells over time, for different reporter strains in minimal glucose + amino-acid medium (M9CGLU). Growth phases are indicated. Data for each reporter were normalized by its mean value. Lines are guides to the eye, except for the katG reporter, in which the data was smoothed by a Gaussian low-pass filter. Error bars indicate standard errors. (A) Normalized mean fluorescence of the arabinose system reporters, and katG. (B) Normalized mean fluorescence of ribosomal reporters.

(C) Normalized mean fluorescence of amino-acid genes reporters.

immediately begin lag2 and produce biomass. This can explain why for some sugars, such as glucose, lag1 phase is not observed. If there are sufficient glucose utilization 


\section{(I) Optimal control model} $u(t)=$ fraction of resources devoted for biomass production $(M)$.

$1-u(t)=$ fraction of resources devoted for production of bottleneck protein $(P)$.

The extended Baranyi's model [19]:

$$
\begin{aligned}
& \frac{d P}{d t}=(1-u) v P \\
& \frac{d M}{d t}=u \mu \frac{P}{1+P} M
\end{aligned}
$$

We optimize $u(t)$ for the fastest possible cell doubling in the new medium (minimal time to doubling biomass), given an initial enzyme levels $P_{0}$.

(II) The optimal solution is 'bangbang' control

$u(t)=0$ till time $t=\tau$, then $u(t)=1$.

The optimal lag1 phase duration is:

$$
\tau=\frac{1}{v} \ln \left(\frac{v}{v_{C}}\right), v_{C}=\frac{\mu P_{0}}{\ln (2)}
$$

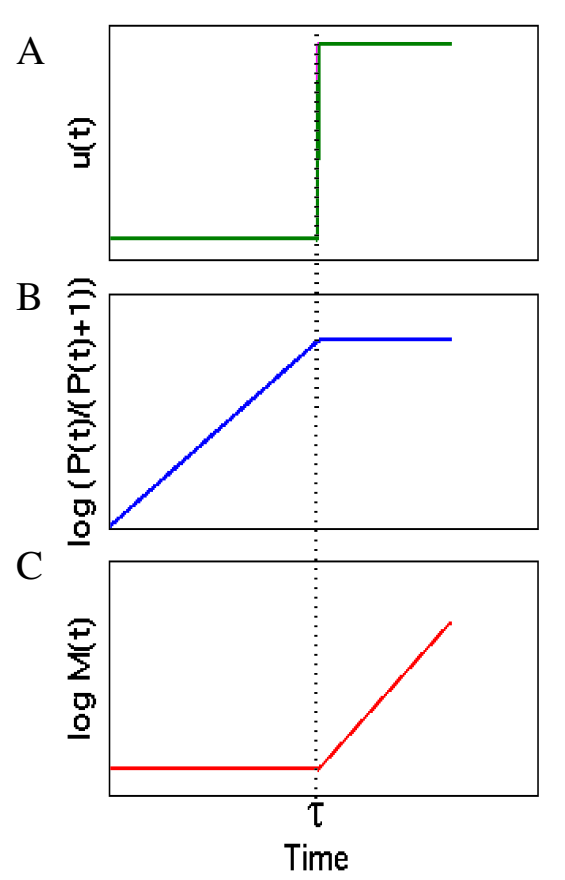

D

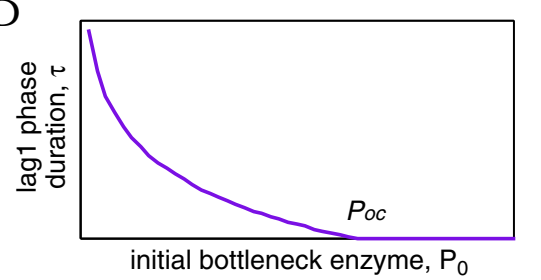

$\mathrm{E}$

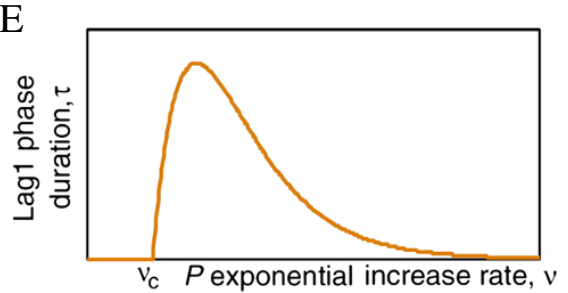

Figure 5 An optimal control model suggests a sudden shift from making bottleneck proteins to full biomass production. (A) The model seeks the optimal allocation function $u(t)$ between making bottleneck protein $P$ and making biomass $M$, in order to minimize the time to double the biomass. The function $u(t)$ is the fraction of resources devoted to making $M$, and $1-u(t)$ the fraction devoted to making $P$. The rate of production of $P$ is parameterized by a maximal rate $v$. The maximal rate of producing $M$ is the exponential growth rate $\mu$. A logistic term is omitted for simplicity, and including it does not change the qualitative result. The optimal solution for $u(t)$, obtained in the Methods section, is a sharp switch from $u=0$ to a value of $u=1$, at time $\tau$. Lag 1 is the time up to time $\tau$, in which the cells make only $P$. In lag 2 and exponential phases, the period of time after $T$, the cells make both bottleneck protein $P$ and biomass $M$. (B) The level of the bottleneck protein $P$. See Additional file 1: Figure S3. (C) The level of the biomass M. (D) Lag1 phase duration $\tau$, as a function of initial bottleneck protein concentration $P$. (E) Lag 1 phase duration $(\tau)$, as a function of $P$ exponential increase rate $v$.

enzymes in the initial cells, it is better to skip lag1 and begin growth immediately.

The model has three parameters, two of which can be estimated directly from the data: the exponential growth rate $\mu \sim 0.8 \mathrm{hr}^{-1}$ and the bottleneck protein exponential increase rate $v \sim 0.5 \mathrm{hr}^{-1}$. From these, and the observed duration of lag1 phase, $\tau \sim 3 \mathrm{hr}$, one can estimate the initial bottleneck enzyme level in the arabinose experiment: using the formula $\tau=\frac{1}{v} \operatorname{In}\left(\frac{v \mu}{P_{0} \operatorname{In}(2)}\right)$, indicating a low initial enzyme concentration of $P_{O} \sim 0.1$ (in units of its effective Michaelis-Menten constant).

Finally, the model also suggests how different individual cells might vary in their lag1 phase duration. It is known 
that individual cells differ in the concentration of any given protein, an effect known as protein expression noise [22-25]. Cell-cell variations in the initial bottleneck enzyme level $P_{O}$ will thus result in different lag1 durations. Usually, the distribution of protein concentrations across cells is well described by a Gamma distribution [47], which resembles quite closely a log-normal distributions when protein copy number is not too low. Indeed, our flow cytometry measurements of the $\operatorname{araB}$ reporter strain show approximately log-normal distributions at early times (SI, Additional file 1: Figure S2). Because lag phase duration goes as the logarithm of $P_{0}$, the model predicts that cell-cell variation in lag duration will roughly follow a normal distribution (because the lognormal distribution of $P_{O}$ mean a normal distribution of $\ln \left(P_{0}\right)$ ). This prediction agrees with observed distributions of lag times $[26,48]$.

\section{Discussion}

We found that the seemingly inert lag1 phase shows exponentially increasing activity of a few specific promoters. To observe this, we developed an assay using $E$ coli reporter strains, which were imaged during flow cytometry, to measure promoter activity in lag phase. Using this experimental system, we were able to follow cell size and promoter activity dynamics over the bacterial growth curve. This allowed clear distinction between the two lag sub-phases: lag1 and lag2.

With arabinose as the carbon source, we found that gene expression in lag1 phase (the phase where biomass does not grow) is very different from gene expression in exponential phase and lag2 phase (the phase in which biomass grows but not cell number). It is also different from expression in stationary phase before inoculation. In lag1, the genes needed to utilize the specific carbon source in the medium (arabinose) are expressed with exponentially increasing dynamics, despite the fact that biomass does not measurably increase. The exponential rate of expression in lag 1 phase is nearly 2 -fold slower than the exponential rate of cell growth in exponential phase. Utilization genes for other sugars are not expressed. General growth genes such as ribosomal genes are not expressed in lag1 phase, and begin to be expressed only in lag2 when cell biomass increases. Amino acid biosynthesis genes are also not expressed in lag1 phase on arabinose. In a screen of 140 diverse promoters, the only other promoter that rises sharply above its initial level is the oxidative stress promoter $k a t G$. The resulting picture resembles the engineering principle of bang-bang control [49-51], in which resources are devoted exclusively at first to the bottleneck proteins to provide carbon and energy for growth, and only later, at lag2, expression switches to a full growth program [52].

One may interpret the exponential rise in expression of utilization genes in lag1 phase by considering a positive feedback process. After inoculation from stationary phase, the cell contains very few transporters and utilization genes for sugars such as arabinose. The influx of arabinose into the cell is low, and ribosomal production is prevented by the stringent response system [53-56]. The cells seem to utilize their meager initial resources [57] to primarily make new utilization genes: arabinose transporters and catabolic enzymes. In turn, due to these transporters and catabolic genes, arabinose influx increases, expression increases in turn, and an auto-catalytic process occurs. The exponential rate of this process is presumably determined by the rate constants of the transporters and enzymes, and the elongation rate of the translation machinery at low nutrient levels. When the cell has enough amino acids to unlock stringent response, ribosomal production ensues together with biomass growth and exit from lag1 (this is in line with the finding of no apparent effect of a $\triangle r e l A \Delta s p o T$ double deletion on lag2 in rich medium [34], suggesting that ppGpp control of ribosome production is released in lag2).

A very short lag1 phase is expected to be found in fresh media in which cells already express the relevant utilization genes at high levels in stationary phase. For these sugars, such as glucose in the present study, the cell comes prepared for almost immediate growth. It would be of interest to study the evolutionary circumstances that lead cells to choose in which sugar systems to invest during stationary phase.

These findings relate also to theoretical models for lag phase dynamics, such as Baranyi's model [19], which are widely used in the food safety field to estimate lag phase duration in a given food product. Baranyi's model assumes that the cells in lag1 produce a set of enzymes $P$ at an exponential rate $v$, which provide building blocks for biomass production. The present findings may be interpreted as identifying the enzymes $P$ as the sugar utilization genes (ara genes in the present arabinose medium). In many implementations of Baranyi's model to describe population growth curves, it is assumed for simplicity that the exponential rise in the enzymes $P$ occurs at the same rate as the later exponential increase in biomass denoted $\mu$ (that is, that $\nu=\mu$ ) $[19,58]$. The present finding suggest that in the case of arabinose, $P$ rises exponentially but at a different (slower) rate than the exponential rise in cell number $(\nu<\mu)$. This finding can lead to refinements of modeling for better predictions of lag phase duration.

The abrupt switch from lag1 with its specific expression program to lag2 with its general growth program may be interpreted in light of optimal control theory. Under a wide range of conditions, in order to achieve the greatest final product (cells), it is optimal to first make only the limiting resources in lag1 (bottleneck proteins that utilize the carbon source), and then abruptly switch to making all of the biomass production machinery in lag2 (Figure 5). Mechanistically, this switch might be implemented by the 
unlocking of stringent control. A similar strategy is found in wasps that need to allocate resources between making workers and making reproductive individuals [49-51]. Wasps first make only workers, which bring in nutrients, and then, a few weeks before winter, switch to making reproductives (rather than making both workers and reproductives from the onset at a given ratio). Another example is found in development of the mouse gut, where stem cells make an abrupt transition between symmetric and asymmetric divisions, which has been suggested to be the fastest way to reach the desired number of differentiated cells [59].

Future work can extend the present approach to more genes, to fully map the expression strategy in lag phase. It would be important to develop further methods to measure protein quantities required to start the division cycle, in order to develop mechanistic models for bacterial growth phases. Testing the role of stringent response in lag1 phase, and evolutionary experiments to change lag phase duration [60] can help identify genes and regulation important for this process. Another interesting topic is the role of cell-cell variation in lag phase: stochastic effects will result in different individual bacteria making the bang-bang switch at different times [26]. Such studies will require following individual cells and their promoter activity over time.

\section{Conclusions}

In this study we developed a method to explore E. coli gene expression in lag phase. We distinguish between lag1 phase in which there is no visible growth, and lag2 in which cells grow but have not yet divided. When arabinose is the sole carbon source, the vast majority of promoters are inactive in lag1, except for the promoters for the carbon source in the medium (arabinose) which are activated with exponentially increasing dynamics. In lag2, E. coli switches abruptly to a full growth program of promoter activity. This behavior is consistent with a bang-bang control strategy of allocating resources to making bottleneck components first in order to optimize biomass production over time.

\section{Methods}

\section{Strains and plasmids}

E. coli GFP reporter strains (K12 MG1655, with a pUA66 based reporter plasmid, $\mathrm{sc} 101$ ori, $\mathrm{kan}^{\mathrm{R}}$, with the gfpmut 2 gene [61]) are from the fluorescent reporter library described in [35]. Strain U66 with a promoterless reporter plasmid was used for fluorescence background measurements $[35,37,62]$. All strains are available from Open Biosystems (Thermo Fisher Scientific Molecular Biology, USA).

\section{Growth conditions and samples preparation}

The variability in the history of bacterial populations can lead to variability in lag phase behavior even within the same strain [63-65]. We thus employed procedures to reduce lag phase variability between biological repeats and within each experiment. Media was filter sterilized using 0.2- $\mu$ m pore size filters (FP 30/0,2 CA-S, Whatman, UK; and Fast PES filter unit, Nalgene, Thermo Fisher Scientific, USA) to reduce false-positive events in the flow cytometry experiments, and to avoid autoclave steps that can introduce variations in $\mathrm{pH}$ and in other factors to the medium $[34,66,67]$. The same batches of medium constituents were used in all experiments to minimize experimental error. All liquid handling and incubation steps were carried out using a robotic liquid handler (Freedom Evo, Tecan, Switzerland) and incubator (StoreX STX44 ICBT, Liconic Instruments, Liechtenstein) system. Incubations with shaking $(80 \mathrm{~Hz}=$ $4800 \mathrm{RPM}$ ) were carried out at $37^{\circ} \mathrm{C}$. Strains from frozen stocks were pre-grown for 48 hours in u-shaped bottom $2 \mathrm{ml}$ 96-deepwell plates (PlateOne, USA Scientific, USA), with $600 \mu \mathrm{l}$ M9 minimal medium containing $0.2 \%$ glucose, $0.05 \%$ casamino acids, and $50 \mu \mathrm{g} / \mathrm{ml}$ kanamycin. This relatively long incubation time ensures that the cells are in stationary phase long enough to reduce the effects of cell history, and leads to a sizable lag phase. Longer incubation times increase the lag phase cell-to-cell variability presumably due to increased stress [20,26,48,63,64,68]. We controlled for different amounts of time spent in stationary phase pre-inoculation, finding that this variable had little effect on the present conclusions.

The deep well plates were covered with aerationpermitting lids (Universal Lid, Seahorse Bioscience, USA), to reduce contamination and evaporation. The experimental deep well plates were prepared with M9 minimal medium (final volume $500 \mu \mathrm{l}$ ) containing $50 \mu \mathrm{g} /$ $\mathrm{ml}$ kanamycin and $0.2 \%$ of a certain carbon source (glucose; L-arabinose). The glucose medium also contained 0.05\% casamino acids (Bacto, BD, USA).

During 15 hours, every 5-180 minutes, a different column was inoculated with 8 reporter strains at a 1:750 dilution from the stationary phase culture. After 15 hours, the deep well plates contained cells that were from $5 \mathrm{mi}$ nutes up to 15 hours in fresh medium. This conveniently generated a full time-course of growth in one plate, that could be analyzed in a single flow cytometry session. One column in each plate was not inoculated and was used for both medium contamination detection and blank sample measurements. Reporter strains from the stationary phase cultures were diluted 1:750 into pre-cooled M9, and represented the strains at $\mathrm{t}=0$ (end of stationary phase or beginning of lag phase). After the experimental incubation, the cells were rapidly cooled to $4^{\circ} \mathrm{C}$, diluted according to their density (from no dilution at all, up to 1:100 dilution), to avoid coincidence in the flow cytometry instruments $[69,70]$, and transferred into pre-cooled u-shaped 96-wells plates (Costar, Corning, USA) for the automated flow cytometry analysis. All samples were measured at 
most 4 hours after the growth experiments ended. The same samples were analyzed both using the LSRII and the ImageStream $^{\mathrm{X}}$ instruments.

No fixation or staining were used as these procedures potentially affect the side scatter (SSC), forward scatter (FSC), and GFP values of bacterial cells, as well as the bacterial count [70-72]. Moreover, the added steps necessary for these procedures might increase variability between the samples and between the experiments.

\section{Flow cytometry measurements \\ Standard flow cytometry}

$20 \mu \mathrm{l}$ sample at a flow rate of $1 \mu \mathrm{l} / \mathrm{s}$ were analyzed per sample using a LSRII (BD, USA) flow cytometry instrument (settings: FSC $648 \mathrm{mV}$, SSC $346 \mathrm{mV}$, GFP $450 \mathrm{mV}$, threshold collection FSC > 200) at $4^{\circ} \mathrm{C}$. The cells' FSC, SSC and GFP values were collected. The events rate for the bacterial samples was between 1,000-9,500 events/s, and did not come near the upper limit of the instrument $(20,000$ events/s), to avoid coincidence [69,70]. A total of 20,000190,000 events were collected per sample. Blank samples with medium only were used in each experiment to exclude contaminations, and to calculate background event numbers, with an average event rate of 50 events/s (Additional file 1: Figure S5). FSC \& SSC were used for gating. In contrast to most flow cytometry experiments that usually use narrow gating to achieve measurements of a homogenized population, a wide gating was used in this study (Additional file 1: Figure S6). This large gating allowed measuring the entire population, and especially to monitor cell size and cell number. The number of background events in the gated area was negligible, between $0.3-3 \%$ of the total number of gated events per sample. The mean day-to-day error between fluorescence measurements for the arabinose system reporters was 9\%, and below $17 \%$ for cell count.

Bacterial growth curves were calculated by the following procedure: In each well, the same liquid volume and liquid flow rate were used to count the number of events (cells) [73]. Combined with the known dilution coefficients (from the original experimental deep well plates), we calculated bacterial cell counts at each time point for each reporter strain.

\section{Imaging flow cytometry}

Around 4000 events were collected per sample using the ImageStream $^{\mathrm{X}}$ imaging flow cytometry instrument and Inspire software (Amnis, Seatle, USA). The cells' bright field (BF), SSC \& GFP images were taken at an event rate of $5-500$ cells/s. The instrument uses beads to track the cell flow and to focus on the cells. Bead images were ignored during image acquisition or removed from the analysis to increase image analysis accuracy. Instrument settings for image collection: raw_max_pixel_SSC $<200$ (ignored most of the bead images), raw_max_pixel_GFP $<4094$ (ignored saturated GFP images), raw_min_area_BF>1 square $\mu \mathrm{m}$ ( $>9$ pixels, this removed small objects suspected as dust and cell fragments). Image analysis was performed using ImageStream $^{\mathrm{X}}$ analysis software IDEAS 4.0 (Amnis, Seatle, USA). Gating: more beads were removed by gating with mean_pixel_BF and mean_pixel_SSC, unfocused cells were removed by gating using gradient_RMS_BF, and more beads were removed by gating with aspect_ratio_intensity_BF and min_pixel_BF [74]. Flow aligns the cell long axis with the flow direction, so that length measurements in $2 \mathrm{D}$ images are an accurate indication of true length [75,76]. Measurements of mean cell length had a mean day to day error of $3 \%$.

Standard flow cytometry and imaging flow cytometry analysis were conducted on the same samples. All experiments were repeated at least twice.

\section{Optimal control model}

We model the lag phase enzyme dynamics using the framework of Baranyi and Roberts [19]. The cells make biomass $M$ at a rate that depends on a bottleneck enzyme (representing for example transporters and catabolic enzymes for arabinose utilization) whose concentration is $P$. The rate of biomass production is dependent on $P$ in a Michaelis-Menten form:

$$
\frac{d M}{d t}=u \mu \frac{P}{(1+P)} M
$$

Where $\mu$ is the maximal growth rate, and $P$ is given in units of its effective halfway concentration, so that at $P=1$ biomass production is half-maximal. The function $u(t)$ is the proportion of resources that the cell devotes to biomass production, which can vary between one and zero.

The bottleneck enzyme $P$ is produced at rate $v P$, times the fraction of resources the cell devotes to it, namely $1-u$ :

$$
\frac{d P}{d t}=(1-u) v P
$$

The objective in the optimization problem we study is to divide rapidly in the new condition. We thus seek the control profile $u(t)$ that will double the biomass in the minimal amount of time, $T$. Initial conditions are $P(t=0)=P_{0}$, the amount of enzyme present in the inoculated cells, and initial biomass $M(t=0)=M_{O}$.

According to Pontryagin's maximum principle $[45,46]$, such minimal time problems are solved by the profile $u(t)$ that maximizes at every time point the Hamiltonian:

$$
\begin{aligned}
H & =-1+\lambda_{1} \frac{d M}{d t}+\lambda_{2} \frac{d P}{d t} \\
& =-1+\lambda_{1} u \mu \frac{P}{(1+P)} M+\lambda_{2}(1-u) v P
\end{aligned}
$$


The Lagrange multipliers $\lambda_{1}(t)$ and $\lambda_{2}(t)$ follow Hamilton's equations:

$$
\frac{d \lambda_{1}}{d t}=-\frac{\partial H}{\partial M}, \frac{d \lambda_{2}}{d t}=-\frac{\partial H}{\partial P}
$$

Because the Hamiltonian $H$ is linear in $u$ (Eq 3), it can be maximal only at the extreme values that $u$ can take, namely zero and one. This means bang-bang control: devote all the cell resources to either $M$ or $P$, with sharp switches between these states allowed. Bang-Bang control is expected with any Hamiltonian function that is linear or convex in $u$, a very wide class of possible models [46]. Analyzing the equations for $\lambda_{1}$ and $\lambda_{2}$ shows at most a single switch between the extreme values of $u$ can occur in the present model.

Assuming a single switch between making only $P$ and then switching to make only $M$, with switching at time $\tau$, one can solve Eq 2 with $u=0$ to find

$$
P(\tau)=P_{0} e^{v \tau}
$$

From time $\tau$ to the division time $T$, one has $u=1$, so $P$ is constant $=P(\tau)$ and $M$ increases exponentially:

$$
M(T)=M_{0} e^{\mu \frac{(P(\tau))}{(1+P(\tau))}(T-\tau)}
$$

We seek the time $T$ where biomass doubles, $M(T)=2$ $M_{0}$. Solving for $T$ results in

$$
T=\tau+\frac{\operatorname{In}(2)}{\mu}\left(1+\frac{1}{P_{0} e^{v \tau}}\right)
$$

Finding the lag duration $\tau$ that minimizes the time to first division $T$, namely $\mathrm{d} T / \mathrm{d} \tau=0$, results in the following solution:

$$
\tau=\frac{1}{v} \operatorname{In}\left(\frac{v}{v_{c}}\right)
$$

where

$$
v_{c}=\frac{\mu P_{0}}{\operatorname{In}(2)}
$$

Thus, $\tau$ is positive only when $v>v_{c}$. It is equal to zero for $v<v_{c}$, meaning no lag1 phase and a direct transition to lag2 (Figure 5E). The lag phase duration $\tau$ is maximal when $v=e \cdot v_{c}$, where $e=2.71$. Another way to consider this result is that for a given $v$ and $\mu$, there is a critical initial enzyme level $P_{O}$ above which there is no lag1: $P_{O C}=\ln$ (2) $v / \mu$. For the present arabinose parameters, $P_{O C} \sim 0.4$. The duration of lag2 in this model is $\log (2) / \mu+1 / \nu \sim 3 \mathrm{~h}$, which is shorter than the observed arabinose lag2 phase duration, $\sim 5 \mathrm{~h}$. This discrepancy might be explained by using a stochastic model in which lag phase differs between cells. Note that the experimental data (Figure 3) shows increases of total $P$ in lag2 (fluorescence of the ara promoters), whereas the model shows that $P$ concentration becomes constant at lag2. These two observations are consistent, because biomass grows in lag2, and therefore constant $P$ concentration means an increase in total $P$ protein as observed in the experiment.

\section{Additional files}

Additional file 1: Supplemental information. Supporting figures and supporting tables.

Additional file 2: Screen data. 140 promoters normalized screen data. Additional file 3: Screen plots. 140 promoters normalized screen data plots.

\section{Abbreviations}

BF: Bright field; CFU: Colony forming unit; FC: Flow cytometry; FSC: Forward scatter; GFP: Green fluorescent protein; M9CGLU: M9 + 0.05\% casamino acids + 0.2\% glucose minimal medium; M9ARA: M9 + 0.2\% arabinose minimal medium; OD: Optical density; SSC: Side scatter.

\section{Competing interests}

The authors declare that they have no competing interests.

\section{Authors' contributions}

$D M, A B$ and $U A$ designed research; DM, ED and AZ conducted research; DM, $E D, Z P$ and UA analyzed data; DM, ED and UA wrote the paper. All authors read and approved the final manuscript.

\section{Acknowledgments}

We thank our anonymous referees that helped us to finalize the manuscript We thank J. Baranyi and R. Milo for helpful discussions and insightful comments. We thank all of our group members for their support. This work was supported by the Israel Science Foundation, and the European Research Council under the European Union's Seventh Framework Programme (FP7/ 2007-2013) /ERC Grant agreement 249919. The funders had no role in study design, data collection and analysis, decision to publish, or preparation of the manuscript.

\section{Author details}

'Department of Molecular Cell Biology, Weizmann Institute of Science, Rehovot 76100, Israel. ${ }^{2}$ Biological Services Unit, Weizmann Institute of Science, Rehovot 76100, Israel.

Received: 2 May 2013 Accepted: 21 November 2013 Published: 30 December 2013

\section{References}

1. Müller M: Ueber den Einfluss von Fieber temperaturen auf die Wachstumsgeschwindigkeit und die Virulenz des Typhus Bacillus. Z Hyg Infektionskr 1895, 20:245.

2. Penfold WJ: On the nature of bacterial lag. J Hyg (Lond) 1914, 14:215-241

3. Monod J: The growth of bacterial cultures. Annu Rev Microbiol 1949, 3:371-394.

4. Swinnen IAM, Bernaerts K, Dens EJJ, Geeraerd AH, Van Impe JF: Predictive modelling of the microbial lag phase: a review. Int J Food Microbiol 2004 94:137-159.

5. Koyuncu S, Andersson MG, Häggblom P: Accuracy and sensitivity of commercial PCR-based methods for detection of salmonella enterica in feed. Appl Environ Microbiol 2010, 76:2815-2822.

6. Van Impe J, McMeekin T, Olley J, Ratkowsky D: 3rd international conference on predictive modeling in foods. Int J Food Microbio/ 2002, 73:107-454.

7. Bättig P, Hathaway LJ, Hofer S, Mühlemann K: Serotype-specific invasiveness and colonization prevalence in Streptococcus pneumoniae correlate with the lag phase during in vitro growth. Microbes Infect 2006, 8:2612-2617.

8. Hathaway LJ, Brugger SD, Morand B, Bangert M, Rotzetter JU, Hauser C, Graber WA, Gore S, Kadioglu A, Mühlemann K: Capsule type of 
Streptococcus pneumoniae determines growth phenotype. PLOS Pathog 2012, 8:e1002574.

9. Frimodt-Møller N, Sebbesen O, Frølund Thomsen V: The pneumococcus and the mouse protection test: importance of the lag phase in vivo. Chemotherapy 1983, 29:128-134.

10. Baranyi J, George SM, Kutalik Z: Parameter estimation for the distribution of single cell lag times. J Theor Biol 2009, 259:24-30.

11. Buchanan RL, Cygnarowicz ML: A mathematical approach toward defining and calculating the duration of the lag phase. Food Microbio/ 1990, 7:237-240.

12. Buchanan RL, Solberg M: Interaction of sodium nitrate, oxygen and ph on growth of staphylococcus aureus. J Food Sci 1972, 37:81-85.

13. McKellar RC, Knight K: A combined discrete-continuous model describing the lag phase of Listeria monocytogenes. Int J Food Microbiol 2000, 54:171-180.

14. Pirt SJ: Principles of microbe and cell cultivation. New York: Wiley; 1975.

15. Zhou K, George SM, Métris A, Li PL, Baranyi J: Lag phase of salmonella enterica under osmotic stress conditions. Appl Environ Microbiol 2011, 77:1758-1762.

16. Zwietering $\mathrm{MH}$, Rombouts FM, van 't Riet $\mathrm{K}$ : Comparison of definitions of the lag phase and the exponential phase in bacterial growth. J Appl Bacteriol 1992, 72:139-145.

17. Baranyi J, Roberts TA, McClure P: A non-autonomous differential equation to modelbacterial growth. Food Microbiol 1993, 10:43-59.

18. Srivastava AK, Volesky B: Characterization of transient cultures of clostridium acetobutylicum. Biotechnol Prog 1990, 6:408-420.

19. Baranyi J, Roberts TA: A dynamic approach to predicting bacterial growth in food. Int J Food Microbiol 1994, 23:277-294.

20. Pin C, Baranyi J: Kinetics of single cells: observation and modeling of a stochastic process. Appl Environ Microbiol 2006, 72:2163-2169.

21. Métris A, Le Marc Y, Elfwing A, Ballagi A, Baranyi J: Modelling the variability of lag times and the first generation times of single cells of E. coli. Int J Food Microbiol 2005, 100:13-19.

22. Elowitz MB, Levine AJ, Siggia ED, Swain PS: Stochastic gene expression in a single cell. Science 2002, 297:1183-1186.

23. Locke JCW, Young JW, Fontes M, Jiménez MJH, Elowitz MB: Stochastic pulse regulation in bacterial stress response. Science 2011, 334:366-369.

24. Kaern M, Elston TC, Blake WJ, Collins JJ: Stochasticity in gene expression: from theories to phenotypes. Nat Rev Genet 2005, 6:451-464.

25. Golding I, Paulsson J, Zawilski SM, Cox EC: Real-time kinetics of gene activity in individual bacteria. Cell 2005, 123:1025-1036.

26. Elfwing A, LeMarc $Y$, Baranyi J, Ballagi A: Observing growth and division of large numbers of individual bacteria by image analysis. Appl Environ Microbiol 2004, 70:675-678

27. Larsen N, Boye M, Siegumfeldt H, Jakobsen M: Differential expression of proteins and genes in the Lag phase of lactococcus lactis subsp. Lactis grown in synthetic medium and reconstituted skim milk. Appl Environ Microbiol 2006, 72:1173-1179.

28. Cuny C, Lesbats M, Dukan S: Induction of a global stress response during the first step of Escherichia coli plate growth. Appl Environ Microbiol 2007, 73:885-889.

29. Novotna J, Vohradsky J, Berndt P, Gramajo H, Langen H, Li X-M, Minas W, Orsaria L, Roeder D, Thompson CJ: Proteomic studies of diauxic lag in the differentiating prokaryote Streptomyces coelicolor reveal a regulatory network of stress-induced proteins and central metabolic enzymes. Mol Microbiol 2003, 48:1289-1303.

30. Osuna R, Lienau D, Hughes KT, Johnson RC: Sequence, regulation, and functions of fis in salmonella typhimurium. J Bacteriol 1995, 177:2021-2032.

31. McKellar RC: Correlation between the change in the kinetics of the ribosomal RNA rrnB P2 promoter and the transition from lag to exponential phase with pseudomonas fluorescens. Int J Food Microbio 2008, 121:11-17

32. McKellar RC: Effect of starvation on expression of the ribosomal RNA rrnB P2 promoter during the lag phase of pseudomonas fluorescens. Int J Food Microbiol 2007, 114:307-315.

33. McKellar RC: Effect of sub-lethal heating and growth temperature on expression of the ribosomal RNA rrnB P2 promoter during the lag phase of pseudomonas fluorescens. Int J Food Microbiol 2007, 116:248-259.

34. Rolfe MD, Rice CJ, Lucchini S, Pin C, Thompson A, Cameron ADS, Alston M, Stringer MF, Betts RP, Baranyi J, Peck MW, Hinton JCD: Lag phase is a distinct growth phase that prepares bacteria for exponential growth and involves transient metal accumulation. J Bacterio/ 2012, 194:686-701.

35. Zaslaver A, Bren A, Ronen M, Itzkovitz S, Kikoin I, Shavit S, Liebermeister W, Surette MG, Alon U: A comprehensive library of fluorescent transcriptional reporters for Escherichia coli. Nat Methods 2006, 3:623-628.

36. Kaplan S, Bren A, Zaslaver A, Dekel E, Alon U: Diverse two-dimensional input functions control bacterial sugar genes. Mol Cell 2008, 29:786-792.

37. Madar D, Dekel E, Bren A, Alon U: Negative auto-regulation increases the input dynamic-range of the arabinose system of Escherichia coli. BMC Syst Biol 2011, 5:111.

38. Hudson JA, Mott SJ: Comparison of lag times obtained from optical density and viable count data for a strain of pseudomonas fragi. J Food Saf 1994, 14:329-339.

39. Robinson TP, Ocio MJ, Kaloti A, Mackey BM: The effect of the growth environment on the lag phase of Listeria monocytogenes. Int J Food Microbiol 1998, 44:83-92.

40. Kell DB, Young M: Bacterial dormancy and culturability: the role of autocrine growth factors. Curr Opin Microbiol 2000, 3:238-243.

41. Weichart DH, Kell DB: Characterization of an autostimulatory substance produced by Escherichia coli. Microbiology 2001, 147:1875-1885.

42. Mukamolova GV, Kaprelyants AS, Young DI, Young M, Kell DB: A bacterial cytokine. Proc Natl Acad Sci U S A 1998, 95:8916-8921.

43. Kempner ES, Hanson FE: Aspects of light production by photobacterium fischeri. J Bacteriol 1968, 95:975-979.

44. Turovskiy Y, Kashtanov D, Paskhover B, Chikindas ML: Quorum sensing: fact, fiction, and everything in between. Adv Appl Microbiol 2007, 62:191-234

45. Pontryàgin LS, Boltyanskii VG, Gamkrelidze RV, Mishchenko EF: The mathematical theory of optimal processes. New York: John Wiley; 1962.

46. Alexander RM: Optima for animals. Princeton: Princeton University Press; 1996.

47. Cai L, Friedman N, Xie XS: Stochastic protein expression in individual cells at the single molecule level. Nature 2006, 440:358-362.

48. Levin-Reisman I, Gefen O, Fridman O, Ronin I, Shwa D, Sheftel H, Balaban NQ: Automated imaging with ScanLag reveals previously undetectable bacterial growth phenotypes. Nat Methods 2010, 7:737-739.

49. Ishay J, Bytinski-Saltz H, Shulov A: Contributions to the bionomics of the oriental hornet Vespa orientalis. Isr J Entomol 1967, 2:45-106.

50. Macevicz S, Oster G: Modeling social insect populations II: optimal reproductive strategies in annual eusocial insect colonies. Behav Ecol Sociobiol 1976, 1:265-282

51. Oster GF, Wilson EO: Caste and ecology in the social insects. (Mpb-12). Princeton: Princeton University Press; 1979

52. Shoval O, Sheftel H, Shinar G, Hart Y, Ramote O, Mayo A, Dekel E, Kavanagh $\mathrm{K}$, Alon U: Evolutionary trade-offs, pareto optimality, and the geometry of phenotype space. Science 2012, 336:1157-1160.

53. Cashel M, Kalbacher B: The control of ribonucleic acid synthesis in Escherichia coli. V. Characterization of a nucleotide associated with the stringent response. J Biol Chem 1970, 245:2309-2318.

54. Cashel M, Gentry DR, Hernandez VH, Vinella D: The stringent response. In Escherichia coli \& salmonella typhimurium: cellular \& molecular biology. Volume 1. 2nd edition. Edited by Ingraham JL, Neidhardt FC, Ingraham JL, Neidhardt FC. Washington DC: ASM Press; 1996:1458-1496.

55. Schneider DA, Ross W, Gourse RL: Control of rRNA expression in Escherichia coli. Curr Opin Microbiol 2003, 6:151-156.

56. Bouveret $E$, Battesti $A$ : The stringent response. In Bacterial stress response. 2nd Edition. Edited by Storz G, Hengge R. Washington, DC: ASM Press; 2011.

57. Yamamotoya T, Dose $H$, Tian Z, Fauré A, Toya $Y$, Honma M, Igarashi $K$, Nakahigashi K, Soga T, Mori H, Matsuno H: Glycogen is the primary source of glucose during the lag phase of E. coli proliferation. Biochim Biophys Acta 1824, 2012:1442-1448.

58. Zhou K, George SM, Li PL, Baranyi J: Effect of periodic fluctuation in the osmotic environment on the adaptation of Salmonella. Food Microbiol 2012, 30:298-302.

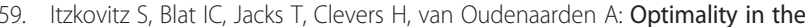
development of intestinal crypts. Cell 2012, 148:608-619.

60. Oxman $E$, Alon $U$, Dekel E: Defined order of evolutionary adaptations: experimental evidence. Evolution 2008, 62:1547-1554.

61. Cormack BP, Valdivia RH, Falkow S: FACS-optimized mutants of the green fluorescent protein (GFP). Gene 1996, 173(1 Spec No):33-38. 
62. Hart Y, Madar D, Yuan J, Bren A, Mayo AE, Rabinowitz JD, Alon U: Robust control of nitrogen assimilation by a bifunctional enzyme in E. Coli. $\mathrm{Mol}$ Cell 2011, 41:117-127.

63. Hershey AD: Factors limiting bacterial growth. J Bacteriol 1939, 37:285-299.

64. Pin C, Baranyi J: Single-cell and population lag times as a function of cell age. Appl Environ Microbiol 2008, 74:2534-2536.

65. D'Arrigo M, de Fernando GDG, Velasco de Diego R, Ordóñez JA, George SM, Pin C: Indirect measurement of the lag time distribution of single cells of listeria innocua in food. Appl Environ Microbiol 2006, 72:2533-2538.

66. Biesta-Peters EG, Mols M, Reij MW, Abee T: Physiological parameters of Bacillus cereus marking the end of acid-induced lag phases. Int J Food Microbiol 2011, 148:42-47.

67. Gennis RB, Stewart V: Respiration. In Escherichia Coli \& Salmonella Typhimurium: Cellular \& Molecular Biology. Volume 1. 2nd edition. Edited by Ingraham JL, Neidhardt FC. Washington, DC: ASM Press:217-261.

68. Guillier L, Pardon P, Augustin J-C: Influence of stress on individual lag time distributions of listeria monocytogenes. Appl Environ Microbiol 2005, 71:2940-2948

69. Marie D, Vaulot D, Partensky F: Application of the novel nucleic acid dyes YOYO-1, YO-PRO-1, and PicoGreen for flow cytometric analysis of marine prokaryotes. Appl Environ Microbiol 1996, 62:1649-1655.

70. Gasol JM, del Giorgio PA: Using flow cytometry for counting natural planktonic bacteria and understanding the structure of planktonic bacterial communities. Sci Mar 2000, 64:197-224.

71. Kamiya E, Izumiyama S, Nishimura M, Mitchell JG, Kogure K: Effects of fixation and storage on flow cytometric analysis of marine bacteria. J Oceanogr 2007, 63:101-112.

72. Günther S, Hübschmann T, Rudolf M, Eschenhagen M, Röske I, Harms H, Müller S: Fixation procedures for flow cytometric analysis of environmental bacteria. J Microbiol Methods 2008, 75:127-134.

73. Monfort P, Baleux B: Comparison of flow cytometry and epifluorescence microscopy for counting bacteria in aquatic ecosystems. Cytometry 1992 13:188-192.

74. George TC, Fanning SL, Fitzgerald-Bocarsly P, Fitzgeral-Bocarsly P, Medeiros RB, Highfill S, Shimizu Y, Hall BE, Frost K, Basiji D, Ortyn WE, Morrissey PJ, Lynch DH: Quantitative measurement of nuclear translocation events using similarity analysis of multispectral cellular images obtained in flow. $\mathrm{J}$ Immunol Methods 2006, 311:117-129.

75. Rajwa B, Venkatapathi M, Ragheb K, Banada PP, Hirleman ED, Lary T, Robinson JP: Automated classification of bacterial particles in flow by multiangle scatter measurement and support vector machine classifier. Cytometry Part A 2008, 73A:369-379.

76. Yamaguchi N, Torii M, Uebayashi Y, Nasu M: Rapid, Semiautomated Quantification of Bacterial Cells in Freshwater by Using a Microfluidic Device for On-Chip Staining and Counting. Appl Environ Microbiol 2011 77:1536-1539.

doi:10.1186/1752-0509-7-136

Cite this article as: Madar et al:: Promoter activity dynamics in the lag phase of Escherichia coli. BMC Systems Biology 2013 7:136.

\section{Submit your next manuscript to BioMed Central and take full advantage of:}

- Convenient online submission

- Thorough peer review

- No space constraints or color figure charges

- Immediate publication on acceptance

- Inclusion in PubMed, CAS, Scopus and Google Scholar

- Research which is freely available for redistribution

Submit your manuscript at www.biomedcentral.com/submit
Biomed Central 